\title{
CLINICAL APPROACH AND GUIDELINES FOR MANAGEMENT OF SEPSIS ASSOCIATED ENCEPHALOPATHY
}

\author{
Ayu Trisnawati ${ }^{1}$, Badrul Munir ${ }^{1}$ \\ Correspondence: emailayutrisna@gmail.com \\ ${ }^{I}$ Department of Neurology Faculty of Medicine Brawijaya University, Malang, Indonesia.
}

\section{Article History:}

Received: August 24, 2018

Accepted: December 11, 2018

Published: July 1, 2019

Cite this as:

Trisnawati A, Munir B. Clinical approach and guidelines for management of sepsis associated encephalopathy. Malang Neurology Journal; 2019.5:98104. DOI:

http://dx.doi.org/10.21776/ub.mnj .2019.005.02.10

\section{ABSTRACT}

Septic associated encephalopathy (SAE) is simply define as an encephalopathy incidence that occurs in sepsis patients. Ironically this entity can increases the mortality rate. Importantly, the clinical presentations of this entity is vary ranging from malaise to coma condition. The central nervous system is susceptible to damage that caused by systemic inflammation including sepsis with complex pathophysiologies such as endothelial dysfunction, neurotransmitter imbalance, oxidative stress, mitochondrial dysfunction and apoptosis. In general, current management of SAE is similar with the management of sepsis incidence which includes infections management, maintaining tissue perfusion and symptomatic therapy to treat delirium and convulsions that may occur. Also, several studies continue to be developed including the use of corticosteroids, magnesium, antioxidants, and immunoglobulin. Early identification and appropriate initial handling significantly affect the output of the SAE patients.

Keywords: Sepsis Associated Encephalopathy, septic condition, brain in sepsis

\section{Introduction}

Sepsis is a life-threatening condition caused by dysregulation of the body's response to infection. Sepsis is considered a world health problem because of the high incidence rate reaches 19 million cases per year, and it has been noted that approximately 750 thousand incidences in America with a mortality rate reaches 1 in 4 event. ${ }^{1}$ Furthermore, sepsis also become the fourth most common cause of delirium in geriatric patients in Saiful Anwar General Hospital, Malang from 2005 to $2010 .^{2}$

The nervous system is susceptible to damage due to a response to systemic inflammation including sepsis. Inflammation induces infiltration of immune cells and inflammatory mediators that affect structural and functional changes. ${ }^{3}$ The term of encephalopathy that related with the $\mathrm{SAE}$ is used to describe the condition of diffuse brain dysfunction. Ironically, this entity occurs because of the infection without a clear sign in the central nervous system (CNS). ${ }^{4,5}$

Furthermore, the encephalopathy in sepsis patients is considered as poor prognosis indicator. This entity leads mortality rate to become double compared to encephalopathy with non-septic causes. ${ }^{3}$ The clinical presentation of SAE varies from mild symptoms such as malaise and concentration impairment to coma condition. SAE is the result of a diagnosis that is enforced after removing other factors that can cause a decrease in consciousness. ${ }^{6}$

In the recent time, there are various theories proposed as the pathogenesis sign of SAE. Brain dysfunction is a result of complex and multifactorial mechanisms such as the excessive circulation of cytokines, a microvascular failure that leads blood-brain barrier (BBB) damage, and mitochondrial dysfunction that causing apoptosis. ${ }^{4,5,6}$

\section{Sepsis Associated Encephalopathy}

\section{Terminology}

By definition, encephalopathy comes from the Latin word "encephalon" which associated with the brain "patheia or pathos" which denotes (a) feeling, (b) disease, (c) treated systemic disease. In general, encephalopathy is defined as a degenerative brain disease. ${ }^{7}$ Commonly encephalopathy caused by the infection in the non-CNS part is known as SAE. By definition, SAE demonstrates brain function disorder that caused by infection in the blood. Another term is known as sepsis-associated delirium that fluctuate consciousness from delirium to coma conditions. ${ }^{7,8}$

On the other hand, the use of septic encephalopathy as the term to determine the SAE condition is still vague. The septic encephalopathy also uses to describe the active infection in CNS. Therefore, the use of septic encephalopathy describes the state of sepsis in response to the infection in the brain or CNS. ${ }^{5}$

\section{Pathophysiology}

The pathophysiology of SAE is almost similar to the basic sign of sepsis where there are many interactions among the microorganism, immune system \& host, and coagulation response. Sepsis occurs when the host's immune response fails to overcome the primary infection. Various factors that interfere this condition such as the characters of 
microorganism, the level of severity caused by infection, superantigens and broad virulence factors, resistance to opsonization, phagocytosis, and the antibiotic. ${ }^{9}$

Sepsis is infected condition (suspected and recorded) that occurs together with systemic manifestations. Moreover, the systemic inflammatory response of sepsis in humans is mainly initiated by components of microbial macromolecules that are not found in human tissue. The most potent molecule that acts as a pathogen-associated molecular pattern (PAMP) is a bacterial lipopolysaccharide (LPS) which known as endotoxin. Importantly, large amounts of PAMP are expressed by Gram-positive bacteria, fungi, parasites, and viruses. This molecule acts as a ligand for receptors expressed in immune effector cells known as Toll-like receptors (TLRs). On the other hand, the Grampositive bacteria do not have endotoxins, but their cell walls are composed of peptidoglycan and lipoteichoic acid which can attach to cell surface receptors and cause inflammation even though they are less effective than LPS. ${ }^{10,11}$

There are several sources of the infection including the environment or nosocomial, where the infection is most often due to pneumonia, followed by intra-abdominal infection, and urinary tract infection. Additionally, the progress of infection also enhances from wounds and infected central venous. Interestingly, the Gram-negative bacteria are commonly found in the blood culture including Escherichia coli, Klebsiella sp., and Pseudomonas aeruginosa then followed by Gram-positive organisms and fungi. The most common Gram-positive bacteria that found in blood culture are Staphylococcus aureus and Streptococcus pneumonia. ${ }^{12}$

The host's defense against infection consists of the innate and adaptive immune system. The innate immune system has quick responds to encounter the microorganism through toll-like receptors (TLRs) recognition. For instance, the TLR-2 recognizes peptidoglycan of Gram-positive bacteria, whereas TLR-4 recognizes lipopolysaccharide of Gramnegative bacteria. TLR bonds in microorganism epitopes stimulate intracellular signals, increasing pro-inflammatory transcription molecules such as tumor necrosis factor- $\alpha$ $(\mathrm{TNF}-\alpha)$, interleukin-1 $\beta$, and interleukin-10. Proinflammatory cytokines increase regulation of adhesion molecules in neutrophils and endothelial cells. Although activated neutrophils can attack microorganisms, they also affect the endothelium through the release of mediators that increase vascular permeability, as well as a release of nitric oxide which acts as a potent vasodilator which causes the septic shock. The coagulation cascade is also initiated by sepsis through activation of endothelial cells to increase the expression of tissue factors, especially factors IIa and VIIIa, which in turn causes microvascular thrombus formation. This condition will strengthen the injury through the release of the mediator and increase distal ischemia and tissue hypoxia due to the occurrence of microvascular obstruction. ${ }^{9}$

Based on pathophysiological approach, there are several sign of encephalopathy in sepsis case which cause brain dysfunction include direct brain cells damage due to mitochondrial dysfunction, endothelial cell damage, neurotransmission disorders, and calcium homeostasis disorder in brain tissue..$^{5}$ Comparatively, the peripheral inflammation contributes to brain cells structure and function change. The infiltration of immune cells and inflammatory mediators toward BBB are toxic and give the direct and indirect negative effect to the brain cells. Neurodegeneration caused by myelin oligodendroglia sheath disorder, also the damage of astrocytes reduce BBB function. Additionally, microgliosis reduce the ability of microglia to phagocyte the apoptotic cells. ${ }^{3}$

Sennoville et al. explained that the peripheral immune system activates two main pathways, the neuronal and the humoral pathway. The neuronal pathway begins with the detection of visceral inflammation by the vagus nerve via axonal cytokine receptors. The secreted-inflammatory product from damaged tissue activates the signal then passed to the nucleus tractus solitarii in the brain stem. As a consequence of the activation of the vagus nerve, the efferent pathway will inhibit the synthesis of cytokines in damaged tissue through the cholinergic anti-inflammatory pathway. The vagus nerve is also connected with other autonomic nuclei, especially those that regulate the adrenal axis and vasopressin secretion. ${ }^{13}$

The second pathway involves the circumventricular organ, the unprotected area from blood-brain barrier and activates the innate and adaptive immune system. Activation of both pathways can activate signals towards the center of behavior, neuroendocrine and neurovegetative. Activation of both pathways activate microglia and increased ROS amount, mediating transcription of pro- and antiinflammatory cytokines in the brain including TNF $\alpha$, IL1 $\beta$, transforming growth factor beta (TGF $\beta$ ), and monocyte chemoattractant protein 1 (MCP1). The mediators modulate the expression of $\alpha$-amino-3-hydroxy-5-methyl-4isoxazolepropionic acid receptors (AMPARs) and $\mathrm{N}$ methyl-D-aspartate receptors (NMDARs) in neurons and cause brain dysfunction. ${ }^{13}$

\section{Disorders of the Neurotransmitter Balance}

Sepsis disrupts neurotransmitters release and expression of cholinergic receptors, beta-adrenergic, gammaaminobutyric acid (GABA) and serotonin. The imbalance that occurs is related to metabolic and inflammatory changes due to sepsis while the NO release the cytokines and prostaglandins modulate beta-adrenergic neurotransmission, GABAergic and cholinergic. This condition mainly occurs in the area of the cortex and hippocampus which plays a role in behavior and emotions. 5,14

During inflammation, including in sepsis, communication occurs between the immune system and the nervous system. Immune cells can affect the synthesis and release of neurotransmitters and express their receptors. Peripheral immune cells have traditionally been divided into innate and adaptive immune systems. The innate immune system includes myeloid cells such as granulocytes, monocytes, macrophages and dendritic cells, and innate lymphocytes such as natural killer cells $(\mathrm{NK}) .^{15}$

Innate immune cells increase rapidly in response to microbes or injury. Cells have identification receptors that are activated by pathogens and cause the release of Damage-associated molecular patterns (DAMP) from epithelial cells to $\mathrm{BBB}$, along with activation of the sympathetic nervous system causing activation of the hypothalamic-pituitary-adrenal axis (HPA) pathway that provides feedback for modulating peripheral inflammation. Because of the immune cells express adrenergic and 
glucocorticoid receptors, they can directly respond to sympathetic nerve signals and activation of the HPA axis that strengthen the inflammatory reflex which also known as a polysynaptic reflex, modulate the inflammatory response and contribute to inflammatory resolution. Dysregulation of this feedback occurs due to changes in glucocorticoid sensitivity. In response to physiological stress and injury due to trauma, neutrophils will increase the number of processes known as granulopoiesis which are then followed by an increase in monocytes. ${ }^{15}$

The parasympathetic system through activation of $\alpha 7$ nicotinic acetylcholine receptors ( $\alpha 7 \mathrm{nAChRs})$ causes a decrease in cytokine synthesis in macrophages and decreases the inflammatory response through stimulation of the vagus nerve or its agonist. Hypocholinergia caused by several mechanisms, namely the disruption of acetylcholine synthesis, and dysfunction due to disturbances in cholinergic synapses including presynapses, synaptic clefts and postsynapses. $5,16,17$

In contrast, catecholamines which are known as neurotransmitters in the sympathetic system will bind to adrenergic receptors that increase the inflammatory response. In the early phase of sepsis, there was an increase in the concentration of catecholamines whereas in septic shock the production and release of endogenous catecholamines decrease and causes inability to maintain the balance of the cardiovascular system. The decrease in catecholamine is probably caused by apoptosis in adrenal medulla cells. ${ }^{16}$

\section{Oxidative Stress, Mitochondrial Dysfunction and Apoptosis}

Oxidative stress promotion in septic conditions occurs in various areas of the brain, especially the hippocampus and cortex. Oxidative stress is induced by cytokines promotion, ROS and nitric oxide which cause mitochondrial damage that results from decreased intracellular antiapoptosis, increased proapoptosis, and reduced adenosine triphosphate (ATP). ${ }^{5,13,14}$

Cell death can also be induced by glutamate through (1) activation of the glutamate N-methyl-D-aspartate (NMDA) receptor subtype, causing excessive increases in $\mathrm{Ca} 2+$ and/or $\mathrm{Na}+$ in neurons; (2) activation of the alpha-amino-3hydroxy-5-methyl-4-isoxazole-propionic acid (AMPA) receptor, causing excessive increases in $\mathrm{Ca} 2+$ and/or $\mathrm{Na}+$ in neurons; and (3) inhibition of cysteine extraction by inducing oxidative stress. ${ }^{14}$

The autopsy results demonstrated that neuronal and microglial apoptosis in patients with septic shock is found in the amygdala, supraoptic and paraventricular nuclei, cervical locus, and autonomic nuclei in the medulla. ${ }^{18}$

\section{Clinical Overview}

The initial symptoms in most patients at the beginning of the sepsis condition are the weakness, anorexia, and malaise \& concentration impairment. In line with the progression of sepsis, patients with mild encephalopathy showed fluctuating states of consciousness and improper behavior. Also, several symptom such as hallucinations and agitation are often seen in the condition of delirium. In line with the progression of sepsis, multiorgan failure will contribute to the severity of encephalopathy with the worst case is coma. ${ }^{5}$ Motoric symptoms such as the rigidity of asterixis paratonia, multifocal tremors and myoclonus can also be found in very limit possibility. ${ }^{14}$

The diagnostic criteria used are based on some symptoms and clinical findings while the diagnosis of SAE is proposed with two main prerequisites, namely the presence of clinical extracranial infections and primary symptoms of impaired consciousness and cognition. SAE can be seen at the beginning of sepsis conditions or the end of the state and is part of multiorgan failure in septic shock conditions. ${ }^{5}$

Nearly $70 \%$ of SAE cases are associated with neuropathy or myopathy or a combination, where the situation is responsible for the failure of weaning in patients using mechanical ventilation. Neuropathy that occurs is axonal damage that requires some duration to repair. ${ }^{5}$

\section{Diagnosis Approach}

SAE diagnosis is a kind of exclusion diagnosis that requires the removal of other possible diagnoses such as central nervous system infections, multi-organ failure, head trauma, fat embolism, and drug side effects. The initial stage in evaluating SAE's suspicion is by assessing the level of awareness and identifying a profile of encephalopathy that can be disorganized with the use of sedation drugs. The next step is to eliminate the primary intracranial lesions that are likely responsible for decreasing the level of awareness experienced. Moreover, the most important thing from identification is finding the source of infection and the pathogen that accountable for causing the sepsis conditions. $^{5}$

A thorough physical examination is needed to identify sources of infection such as pressure sores and cellulitis (inflammatory signs). The absence of bacteremia from the results of culture examination does not exclude the SAE diagnosis because of certain conditions such as previous antibiotic use which can obscure the selection of organisms or the presence of hidden abscesses. ${ }^{5}$

Simple routine laboratory examinations need to be done to detect and correct other metabolic disorders that can cause delirium, or coma such as hypoglycemia, hypercalcemia, hypo- or hypernatremia. ${ }^{13}$

Further research still needed to discover the laboratoryscale markers. Some biomarkers that are widely investigated as markers of brain injury include diagnosing and monitoring septic encephalopathy are Specific Enolase Neurons (NSE), S100 beta, glial fibrillary acidic protein (GFAP) ${ }^{4,19}$ In severe sepsis conditions and septic shock, an increase in S100 beta and NSE, in addition, Procalcitonin and IL- 6 were positively correlated with the pediatric score of mortality score (PRISM) and can be useful in the assessment of pediatric sepsis. $(4)^{3}$

\section{Radiological Examination}

To the greater extent, the radiological examination aims to eliminate the possibility of structural lesions of ischemia, bleeding or other focal lesions in cases where focal deficits are encountered. The radiological profile of SAE has variation results from the normal to abnormalities profile. The most often profile found on magnetic resonance imaging (MRI) examination include various ischemias or white matter lesions and vasogenic edema. In MR angiography profile, vasospasm can occur due to a decrease in blood flow.

\section{Therapeutic Approach}


In the recent time, the primary therapy in sepsis patients including SAE is supportive therapy and overcoming infections that cause sepsis, while encephalopathy-specific treatments are still in various stages of research. The understanding of the causes of encephalopathy is needed to develop therapeutic research. ${ }^{4}$

General sepsis management refers to the recommendation of Surviving Sepsis Campaign in 2016 with the main goal of maintaining tissue perfusion. Other supportive therapies for ICU patients that can be proposed include management of organ failure, avoidance of neurotoxic drugs, and developing preventative strategies to reduce the duration of brain dysfunction. $^{13}$

Other therapeutic approaches such as the use of insulin, activated protein $\mathrm{C}$, and steroids may affect endothelial activation, BBB damage, neuroinflammation, and oxidative stress or apoptosis associated with the pathophysiological mechanism of SAE. But there is no substantial evidence based on a clinical trial that shows the three therapies can reduce the incidence and severity of $\mathrm{SAE}^{14}$ while the sepsis treatments based on the pathophysiologies including eritoran, LPS specific antibodies and TNF, corticosteroids, COX inhibitors and antithrombin is under development stage. $^{20}$

\section{Corticosteroids}

Glucocorticoids (GC) and mineralocorticoids are used as therapies, and it has been known as immunosuppressive, anti-inflammatory, and anti-allergic agent. Over 50 years the dexamethasone has been used to relieve cerebral edema due to tumors by reducing the tight junction permeability of BBB. $^{21}$

The use of corticosteroids is not recommended in septic shock patients who show improvements in hemodynamics after fluid resuscitation and vasopressors, but if the target of hemodynamic improvement is not achieved by fluid resuscitation, the use of intravenous hydrocortisone is recommended at a dose of $200 \mathrm{mg} /$ day. Monitoring of blood sugar levels is needed in sepsis conditions; it is recommended to use insulin in glucose conditions more than $180 \mathrm{mg} / \mathrm{dL}$.

The controversy over corticosteroid use still happening, where corticosteroids are considered to have severe side effects in sepsis including neuromyopathy and hyperglycemia, decreased lymphocytes, immunosuppressed conditions and loss of intestinal epithelial cells due to the occurrence of apoptosis. Immunosuppression conditions cause nosocomial infections and impaired wound healing. ${ }^{9}$

The CORTICUS (Corticosteroid Therapy of Septic Shock) study compared the administration of hydrocortisone to placebo in 126 sepsis patients. In that study, about 63 patients were death at 28 days. A total of 31 patients from 69 people $(45 \%)$ were death in the hydrocortisone group and 32 from 57 patients $(56 \%)$ in the placebo group. These results suggest that there was a difference of $11 \%$ mortality at 28 days. Annane et al., 2011 in a review suggested corticosteroid administration in patients at the start of sepsis conditions using doses more than $0.5 \mu \mathrm{g} / \mathrm{kg} /$ minute. Provision of corticosteroids was continued for five or seven days if there is positive feedback to the administration of hydrocortisone $200 \mathrm{mg} /$ day and fludrocortisone $50 \mu \mathrm{g} /$ day. If vasopressor dependence patients showed negative effect after two days, then corticosteroids administration should be stopped. This dose-based method is according to a placebo-controlled, randomized, double-blind, parallelgroup trial study conducted at 19 ICUs in France for four years in 299 patients who experienced septic shock. From that study, the death incidence were found in first 28 days which accounting about $63 \%$ (73 out of 115 people) in the control group, and $53 \%$ (60 of 114 people) in the corticosteroid group. It was concluded that administration of low doses of hydrocortisone and fludrocortisone for seven days could reduce the risk of death in patients who experience septic shock. ${ }^{22}$

The administration of steroids should be started in the first 24 hours of septic shock; this is based on several studies in experimental animals and clinical trials. Five randomized trials assessed the effect of low to moderate doses of corticosteroids given in the first 24 hours of septic shock, with 118 deaths from 222 patients receiving corticosteroids and 139 deaths from 223 control patients. Moreover, five studies of corticosteroid administration within 72 hours did not affect in reducing mortality. ${ }^{22}$

The other corticosteroids such as methylprednisolone and dexamethasone were used to control sepsis incidence. Glucocorticoids inhibit inflammation through several mechanisms, including: promoting the transcription of I $\mathrm{B} \alpha$ protein $(\mathrm{NF}-\kappa \mathrm{B}$ inhibitor- $\alpha$ ), inhibiting NF- $\kappa \mathrm{B}$ (nuclearfactor $-\kappa \mathrm{B})$ transcription signals; increasing the expression and activation of the annexin 1 protein (also called lipocortin), which inhibits the initial pathways of eicosanoid production such as prostaglandin I2 (PGI2; also known as prostacyclin) and thromboxane A2; and induction of expression of mitogen-activated protein kinase (MAPK) phosphatase 1, which causes inhibition of several proinflammatory pathways. ${ }^{20}$

Based on the Surviving Sepsis Campaign: International Guidelines for Management of Sepsis and Septic Shock in 2016, the use of intravenous hydrocortisone is not recommended for septic shock treatment if the administration of fluid resuscitation and vasopressor is sufficient to restore hemodynamics. Vice versa, if this condition is not reached the standard so that the hydrocortisone (200mg/day) should be intravenously administrated to the patients. ${ }^{1}$

\section{Antioxidants}

Under normal conditions, mitochondria will protect cells from damage caused by ROS through several antioxidant pathways, but when antioxidant protection is experiencing interference, oxidative stress initiating damage at nucleotide acids, proteins and lipids in the mitochondria, which caused loss of enzyme function in the electron transport chain circuit. Therefore mitochondrial dysfunction and disruption of ATP production occur . ${ }^{23}$

Since oxidative damage to mitochondria plays a vital role in the pathology of sepsis, antioxidants can be a potential therapy. However, no studies have provided proper evidence of the benefits of antioxidant supplementation in critical patients. Possibly, this is because the antioxidant distribution in the body does not accumulate in the mitochondria. So that antioxidants are needed which selectively make mitochondria as a target which in turn effectively reduce mitochondrial damage and apoptosis. ${ }^{23}$

A prospective randomized control trial (RCT) study conducted in Tehran from 2012 to 2014 in 54 sepsis 
patients (>17 years) who were given selenium in the form of selenium sodium ( $2 \mathrm{mg}$ IV bolus followed by $1.5 \mathrm{mg}$ for 14 days through continuous infusion) and standardized therapy obtained in 28 days did not provide a significant difference compared to the control group even though the mortality rate was lower at $31 \%$ compare to $40 \%$. The administration of selenium can increase the activity of glutathione peroxidase without effecting inflammatory cytokines such as IL-6, IL-8, and IL-10. ${ }^{24}$

\section{Antagonists of MD2-TLR4}

Eritoran is an antagonist of lipid A synthetic that inhibit LPS bonds on MD2-TLR4 receptors on the cell surface. It has been known that LPS is a major component of the outer membrane of Gram-negative bacteria that triggers inflammation in sepsis. Eritoran is an analogue of lipid A synthetic and specific antagonist of LPS. In phase 1 of study, eritoran blocking the cytokine response in healthy people while in phase 2 of study, eritoran given to critical patients. From this study, there was an insignificant reduction in mortality rate (eritoran $37.5 \%$ versus placebo $56.3 \%)^{25}$

In the phase- 3 study conducted by Opal et al. in 1961, the sepsis patients given tetrasodium eritoran (105 $\mathrm{mg}$ in total) with a final assessment was evaluated from the mortality incidence at 28 days, and the final secondary assessment was assessed from the mortality incidence in months 3,6 and 12 after therapy. In conclusion, this phase- 3 study did not show significant improvement in the output of sepsis and septic shock patients. ${ }^{25}$

\section{Immunoglobulin}

Sepsis is the results from the immune system failure to react against the infection. The pro and anti-inflammatory fluctuation responses usually occur in a healthy person where the source of infection cannot be controlled. Antiinflammation is characterized by a failure of the immune system to respond to bacterial stimuli. At this stage, predominant lymphocytes involving B lymphocytes and subsequently affecting immunoglobulin production. In the development of study related to immunosuppression conditions that occur in sepsis conditions, the concept of stimulating the immune response or replacing critical factors of the immunological system is emerging as a promising new therapeutic strategy. ${ }^{26}$

The biological approach of Intravenous Immunoglobulin (IVIG) therapy in sepsis is generally divided into four categories: (i) playing a pivotal role in the introduction of pathogens, cleansing and act as a scavenger; (ii) carrying and inhibiting transcription of upstream mediator genes such as TNF-alpha, NF- $\kappa \mathrm{B}$ and other proinflammatory cytokines; (iii) bring and inhibit transcription of 'downstream mediator' inflammatory genes such as IFN- $\alpha$, $\beta$ and $-\gamma$, IL- $1 \alpha,-2,-4,-6,-8$, and -10, TNF- $\alpha$ and $-\beta$; (iv) non-apoptotic effects by improving neutrophil function and activating B-cell proliferation. ${ }^{27}$

In several studies, monoclonal antibodies were used such as (i) anti-endotoxin namely E5, anti-lipopolysaccharide (antiLPS); (ii) anti-cytokines namely interleukin-1 (IL-1), antitumor necrosis factor alpha (TNF alpha); and (iii) human monoclonal antibody against Enterobacteriaceae antigen. ${ }^{29}$ While polyclonal immunoglobulin is used with a variety of dosage and administration methods. The standard IVIG of polyclonal antibody dose in neonates firmly starts from 500 $\mathrm{mg} / \mathrm{kg}$ as a single dose in two hours, or a dose of $500 \mathrm{mg} / \mathrm{kg}$ intravenously for four to six hours then repeated in the next 48 hours. Besides, a dose of $1 \mathrm{~g} / \mathrm{kg}$ is also used for three days. The dosage used in IVIG of IgM-enriched is 5 $\mathrm{ml} / \mathrm{kg} /$ day for three days. In adults, the dose of polyclonal IVIG also varies depending on the type of IVIG, starting from $250 \mathrm{mg} / \mathrm{kg}$ for two days, $400 \mathrm{mg} / \mathrm{kg} /$ day for three days, and $1 \mathrm{~g} / \mathrm{kg}$ on the first day followed by $500 \mathrm{mg} / \mathrm{kg}$ on the second and third days. In adults, Ig-M enriched IVIG can be given at a dose of $1300 \mathrm{ml}$ by infusion within 72 hours. $^{28}$

The study conducted by Hamano et al., In 79 ICU patients who experienced sepsis or septic shock given a single dose of IVIG 15 gr. From this treatment, some factor such as procalcitonin, C-reactive protein (CRP), leukocytes, blood lactate, IL-6, and SOFA values were decreased, but there was no significant difference from the prognosis if it was assessed from the mortality rate. ${ }^{29}$

The meta-analysis study was taken from the Cochrane review, MEDLINE, and EMBASE baseline data. This study revealed about 43 randomized controlled trials involving 19,202 patients with sepsis or septic shock until 2012 in 23 countries. This study tried to compare the use of IVIG (monoclonal or polyclonal) with placebo or without intervention. The results of this meta-analysis showed a reduction in mortality in adult sepsis patients given IVIG compared to placebo or without intervention. ${ }^{28}$

This study concluded that polyclonal IVIG reduced mortality in adults with sepsis, but this advantage was not found in several studies that had a low risk of bias values. Whereas polyclonal IVIG used as adjuvant therapy in neonates with sepsis did not show a significant reduction in mortality. Studies on the administration of Ig-M-enriched IVIG in neonates and adults are still small and do not have sufficient evidence to support firm conclusions about the advantage of IVIG administration. Moreover, monoclonal IVIG adjuvant therapy is still in various experimental studies. This result contrasts with several other metaanalysis studies which show that IVIG administration in sepsis patients can provide a benefit in the form of a significant reduction in mortality. ${ }^{28}$

The sepsis guideline from the 2014 Japanese Society of Intensive Care Medicine Committee, the use of immunoglobulin still does not have strong evidence to support better prognosis in sepsis patients and reduce the duration of ventilator use. The IVIG administration that given at the beginning of sepsis conditions provides better results with the recommended dose of $0.2-1 \mathrm{~g} / \mathrm{kg}$ within $\geq$ three days. ${ }^{30}$ While recommendations for Surviving Sepsis Campaign: International Guidelines for Management of Sepsis and Septic Shock in 2016 are not recommended as same as in 2013 with the concern of the use of IVIG in sepsis and septic shock patients. This recommendation considers numerous studies that show the benefits of IVIG administration having a high risk of bias. ${ }^{1}$

\section{Sequelae}

The study conducted by Semmler et al. found cognitive deficits in long-term memory in sepsis rat models, where it was hypothesized as a result of connections disruption between the posterior parietal cortex, medial temporal lobe and prefrontal cortex as well as activation of ascending reticular activating system (ARAS) in the brain stem. ${ }^{4}$ 
The septic shock condition causes some areas of the brain to be susceptible to ischemia such as Ammon's horn, lentiform nucleus, dentate nucleus, hypothalamus, locus coeruleus, amygdala, and autonomic nucleus in the medulla. In the hippocampus area, lesions can occur due to inflammation. The ischemia or hypoxic can explain psychological and cognitive disorders that occur in patients who survive the critical phase. ${ }^{13}$

The study conducted by Semmler et al. 2012 in post-ICU patients in both sepsis and non-sepsis and was followed for 24 months. In this study, an assessment of brain morphology, EEG, and cognitive function was assessed using Neuro-Cognitive Effects (NeuroCogFx) which consisted of evaluation of short-term memory, awareness, attention, verbal memory, figural memory and phonetic verbal fluency. Patients recovering from sepsis showed a decrease in cognitive function without psychiatric disorders. Sepsis survivors showed cognitive deficits in verbal memory, and a significant reduction in hippocampal volume was found on the left side compared to healthy people as controls. In this study, there was permanent cognitive impairment in several domains. ${ }^{31}$

Neuromuscular dysfunction commonly seen in the patients with critical illness myopathy (CIM) that identified as the cause of prolonged weakness. Also, another cause of weakness in critically patients is critical illness polyneuropathy (CIP) which is a general neuropathy and is commonly found in patients with sepsis and multi-organ failure. $^{32}$

CIM is a significant cause of diffuse muscle weakness and patient's weight with the critical condition in ICU. About $50 \%$ of patients who are in the ICU for more than three days have evidence of CIM electrophysiology (+) and CIP $(+)$. Clinically, about $50-70 \%$ of patients who are in the ICU for more than one week will develop CIM (+) and or CIP (+), this number will increase to $100 \%$ in patients who are staying longer in the ICU with sepsis and organ failure. $^{32}$

Based on the examination, the number of Creatinine Kinase (CK) is increased about $50 \%$ with the highest peak in 2-5 days and then gradually back to normal. Nerve conduction studies (NCS) provide a normal sensory response (unless there is CIP), the amplitude of the motor response can be average or decreased. The motor conduction velocity is normal (can slow down if there is a CIP). From EMG examination, the abnormal spontaneous activity (positive waves and fibrillation) was seen in several cases. Short duration and sometimes polyphasic motor units with initial recommendations. Muscle biopsy is the gold standard, but sometimes it is not necessary. ${ }^{32}$

Prevention can be done by minimizing the use of high-dose steroids and neuromuscular blocking agents. Also, aggressive insulin treatment is recommended to maintain blood sugar at $80-110 \mathrm{mg} / \mathrm{dl}$ and can reduce the incidence of CIM and CIP. Early rehabilitation combined with mobilization is a therapeutic and preventive strategy for patients with critical conditions and can improve long-term improvement and functional dependence of patients, and shorten the duration of ventilator use. ${ }^{33}$

CIM with or without CIP occurrence will enhance followed with the increasing of treatment length in the ICU, and the hospital can increase the mortality rate. Spontaneous repair can occur weekly in mild cases and monthly in moderate circumstances. Severe cases may occur with chronic disability and loss of mobilization ability. ${ }^{33}$

\section{Conclusion}

Brain dysfunction that occurs in sepsis conditions has complex pathophysiology including inflammatory and noninflammatory processes that affect all brain cells and induces BBB damage, intracellular metabolic dysfunction, and brain cell death.

As a diagnosis of exclusion, before enforcing SAE it is necessary to investigate other possible etiologies that can still be overcome such as systemic organ dysfunction, bleeding stroke, brain infection or other metabolic disorders, so that a thorough examination is required ranging from a complete neurological examination to a supporting examination such as EEG and brain imaging.

The guideline for the management of SAE includes supportive management to maintain hemodynamic stability, maintain tissue perfusion through optimal oxygenation, and requires adequate treatment of the cause of infection. The use of other supportive therapies such as corticosteroids, magnesium, MD2-TLR4 antagonists, antioxidants and immunoglobulin is still controversial but provides benefits in decreasing ICU treatment duration and ventilator usage, although it has not been proven to be statistically significant in reducing mortality in septic patients. The use of immunoglobulin in sepsis patients is still controversial, where several Meta-analyses denote different results in reducing mortality.

SAE has a poor prognosis with a high mortality rate. Besides, the most frequent sequelae are cognitive and myopathic disorders which can affect the patient's quality of life. The high mortality and morbidity in the form of long-term neurological disorders makes SAE essential to understand and wary because this condition can occur before fulfilling the criteria for sepsis diagnosis such as multi-organ failure.

\section{Acknowledgement}

The authors would like to thank to Brawijaya University and Dr. Saiful Anwar General Hospital, Malang, East Java, Indonesia for facilitating this review.

\section{References}

1. Rhodes A, Evans LE, Alhazzani W, Levy MM, Antonelli M, Ferrer R, et al. Surviving sepsis campaign: international guidelines for management of sepsis and septic shock: 2016. Intensive Care Med. Springer Berlin Heidelberg; 2017.34:304. DOI: 10.1007/s00134-017-4683-6

2. Sunarti S, Rahayu M, Desetyaputra DR. Geriatric patient with delirium profile in saiful anwar general hospital malang from january 2005 until june 2010. Malang Neurology Journal; 2015.1:61-7. DOI: http://dx.doi.org/10.21776/ub.mnj.2015.001.02.3

3. Sankowski R, Mader S, Valdés-ferrer SI. Systemic inflammation and the brain: novel roles of genetic, molecular, and environmental cues as drivers of neurodegeneration. Front Cell Neurosci; 2015.9:1-20. DOI: $10.3389 /$ fncel.2015.00028 
4. Zampieri FG, Park M, Machado FS, Azevedo LC. Sepsis-associated encephalopathy: not just delirium. Clinics (Sao Paulo); 2011.66(10):1825-31. DOI: $10.1590 / \mathrm{S} 1807-59322011001000024$

5. Chaudhry N, Duggal AK. Sepsis Associated Encephalopathy. Adv Med Hindawi Publ Corp. 2014

6. Ropper AH, Samuels MA, Klein JP. Adams and Victor's Principles of Neurology. 10th ed. McGrawHill Education; 2014

7. Albert D, Block AM, Bruce BB, E.Haines D, McCloskey LJ, Mitchell RN, et al. Dorland's illustrated medical dictionary. 32nd ed. Philadelphia: Elsevier Saunders; 2012

8. Zhang Q, Sheng Z, Yao Y. Septic encephalopathy: when cytokines interact with acetylcholine in the brain. Mil Med Res. 2014;1(20):1-9

9. Heming N, Mazeraud A, Verdonk F, Bozza FA, Chrétien F, Sharshar T. Neuroanatomy of sepsisassociated encephalopathy. Critical Care; 2017.21:26. DOI: $10.1186 / \mathrm{s} 13054-017-1643-\mathrm{z}$

10. Opal SM. Endotoxins and other sepsis triggers. Karger; 2010.167:14-24. DOI: 10.1159/000315915

11. Brown L, Wolf JM, Prados-rosales R, Casadevall A. Through the wall: extracellular vesicles in Grampositive bacteria, mycobacteria and fungi. Nat Publ Gr. Nature Publishing Group; 2015.(September). DOI: $10.1038 /$ nrmicro3480

12. Sweis R, Ortiz J, Biller J. Neurology of sepsis. Curr Neurol Neurosci Rep; 2016.16(21):1-10. DOI: 10.1007/s11910-016-0623-Z

13. Sonneville R, Verdonk F, Rauturier C, Klein IF, Wolff M, Annane D. Understanding brain dysfunction in sepsis. Ann Intensive Care; 2013.3(15):1-11. DOI: 10.1186/2110-5820-3-15

14. Dal-Pizzol, Felipe, Tomasi, Cristiane D, Ritter, Cristiane. Septic encephalopathy: does inflammation drive the brain crazy? Brazilian Journal of Psychiatry; Epub June 11, 2014.36(3), 251-258. DOI: $10.1590 / 1516-4446-2013-1233$

15. Hodes GE, Kana V, Menard C, Merad M, Russo SJ. Neuroimmune mechanisms of depression. Nat Neurosci; 2015.18(10). DOI: 10.1038/nn.4113

16. Kanczkowski W, Sue M, Bornstein SR. Adrenal Gland Microenvironment and its involvement in the regulation of stress-induced hormone secretion during sepsis. Front. Endocrinol; 2016. 7:156. DOI: 10.3389/fendo.2016.00156

17. Garzoni L, Faure C, Frasch MG. Fetal cholinergic anti-inflammatory pathway and necrotizing enterocolitis: the brain-gut connection begins in utero. Front Integr Neurosci; 2013.7(August):1-9. DOI: 10.3389/fnint.2013.00057

18. Poujade J, Sonneville R, Souweine B, Azoulay E, Darmon M, Mariotte E, et al. Determinants and prognostic value of sepsis- associated encephalopathy: insights from the prospective multicentre outcomerea registry. Intensive Care Med Exp; 2015.3(Suppl 1):12. DOI: $10.1186 / 2197-425 X-3-S 1-A 48$.

19. Zenaide PV, Gusmao-Flores D. Biomarkers in septic encephalopathy: a systematic review of clinical studies. Rev Bras Ter Intensiva; 2013.25(1):56-62. PMC4031860
20. Fink MP, Warren HS. Strategies to improve drug development for sepsis. Nat Publ Gr. Nature Publishing Group; 2014. DOI: 10.1038/nrd4368

21. Witt KA, Sandoval KE. Steroids and the blood - brain barrier: therapeutic implications. 1st ed. vol. 71, pharmacology of the blood brain barrier: targeting cns disorders. Elsevier Inc; 2014. P. 361-390

22. Annane D. Corticosteroids for severe sepsis: an evidence- based guide for physicians. Ann Intensive Care. Springer Open Ltd; 2011.1(1):7. DOI: 10.1186/2110-5820-1-7

23. Galley HF. Bench-to-bedside review: Targeting antioxidants to mitochondria in sepsis. Gall Crit Care; 2010.14(230). DOI: 10.1186/cc9098

24. Chelkeba L, Ahmadi A, Abdollahi M, Najafi A, Ghadimi MH. The effect of parenteral selenium on outcomes of mechanically ventilated patients following sepsis: a prospective randomized clinical trial. Ann Intensive Care. Springer Paris; 2015.5(29). DOI: 10.1186/s13613-015-0071-y

25. Opal SM, Francois B, Larosa SP, Angus DC, Schein R, Lynn M, et al. Effect of Eritoran, an Antagonist of MD2-TLR4, on Mortality in Patients With Severe Sepsis. J Am Med Assoc; 2015.309(11). DOI: 10.1001/jama.2013.2194

26. Bermejo-martin JF, Giamarellos-bourboulis EJ. International journal of antimicrobial agents endogenous immunoglobulins and sepsis: new perspectives for guiding replacement therapies. Int $\mathrm{J}$ Antimicrob Agents. Elsevier BV; 2015.46. DOI: 10.1016/j.ijantimicag.2015

27. Shankar-hari M, Spencer J, Sewell WA, Rowan KM, Singer M. Bench-to-bedside review: immunoglobulin therapy for sepsis - biological plausibility from a critical care perspective. Crit Care; 2012.16(206). DOI: $10.1186 / \mathrm{cc} 10597$

28. Marissa A, Mad L, Leinolla D, Jb MIII. Intravenous immunoglobulin for treating sepsis, severe sepsis and septic shock (Review). Cochrane Database Syst Rev; 2013.(9). DOI: 10.1002/14651858.CD001090.pub2

29. Hamano N, Nishi K, Onose A, Okamoto A, Umegaki T, Yamazaki E, et al. Efficacy of single-dose intravenous immunoglobulin administration for severe sepsis and septic shock. J Intensive Care; 2013.1(4):23. DOI: $10.1186 / 2052-0492-1-4$

30. Oda S, Aibiki M, Ikeda T, Imaizumi H, Endo S, Ochiai R, et al. The Japanese guidelines for the management of sepsis. J Intensive Care; 2014.2(55). DOI: $10.1186 / \mathrm{s} 40560-014-0055-2$

31. Semmler A, Widmann C N, Okulla T, Urbach H, Kaiser M, Widman G, et al. Persistent cognitive impairment, hippocampal atrophy and EEG changes in sepsis survivors. Journal of Neurology, Neurosurgery \& Psychiatry; 2013.Jan;84(1):62-9. DOI: 10.1136/jnnp-2012-302883

32. Khan J, Harrison TB, Rich MM. Mechanisms of neuromuscular dysfunction in critical illness. Crit Care Clin; 2009.24(1) DOI: 10.1016/j.ccc.2007.10.004

33. Zhou C, Wu L, Ni F, Ji W, Wu J, Zhang H. Critical illness polyneuropathy and myopathy: a systematic review. Neural Regen Res; 2014.9(1):101-10. DOI: 10.4103/1673-5374.125337 